\title{
Computer Recognition of Three-Manifolds
}

\author{
Sergei V. Matveev
}

\section{CONTENTS}

Introduction

1. Special and Almost Special Spines

2. Simplifying Moves on Spines

3. Experimental Results and Conjectures

4. Spines of Manifolds with Essential Annuli

5. The Algorithm

Acknowledgments

References

Research at MSRI was supported in part by the Russian Fond of Fundamental Investigations grant N 96-01-847, by INTAS project $94-921$, and by NSF grant DMS-9022140.
We describe the theoretical background for a computer program that recognizes all closed orientable three-manifolds up to complexity 8 . The program can treat also nonclosed threemanifolds and manifolds of greater complexity, but without necessarily succeeding in recognizing them.

\section{INTRODUCTION}

Let $M$ be an orientable three-manifold such that $\partial M$ is either empty or consists of tori. Then, if we assume Thurston's geometrization conjecture [Scott 1983], $M$ can be decomposed in a unique way into graph-manifolds and hyperbolic pieces. A graph-manifold is a three-manifold that can be obtained by pasting together copies of $D^{2} \times S^{1}$ and $N^{2} \times S^{1}$, where $N^{2}$ is a disc with two holes, along homeomorphisms of their boundaries. In particular, all Seifert manifolds and solv-manifolds can be obtained in this way.

The classification of graph-manifolds is known [Waldhausen 1967a; 1967b], and a list of cusped hyperbolic manifolds up to complexity 7 is contained in [Weeks 1985]. If we possess information on how the pieces are glued together, we can get an explicit description of $M$ as a sum of geometric pieces. Such a presentation is usually sufficient for understanding the intrinsic structure of $M$; it allows one to label $M$ with a name that distinguishes it from all other manifolds.

We describe the theoretical background and outline of a computer algorithm that recognizes threemanifolds, in the following sense: given (by means of combinatorial description, say) a three-manifold $M$ whose boundary is empty or consists of tori, the algorithm attempts to decompose $M$ into geometric pieces and identify those pieces. The algorithm 
always succeeds if $M$ is closed, orientable and has complexity at most 8 (all such manifolds are graphmanifolds; see [Matveev 1990]).

\section{SPECIAL AND ALMOST SPECIAL SPINES}

Definition. A compact polyhedron $P$ is simple if the link of each of its points is homeomorphic to either a circle, a circle with a diameter, or the one-skeleton of a three-simplex (a circle with three radii). Points whose link is not a circle are called singular. (See Figure 1.)

The set of singular points of a simple polyhedron $P$ is called the singular graph of $P$. Each connected component of the complement of the singular graph of $P$ is a two-manifold without boundary, and is called a two-component of $P$.

Definition. A compact polyhedron $P$ is called almost special if it can be embedded in a simple polyhedron.

There is a close relation between simple and almost special polyhedra. For example, the wedge of any simple polyhedron and any graph is an almost special polyhedron. The example is very typical, since any almost special polyhedron can be collapsed onto a polyhedron of the form $P \cup G$, where $P$ is a collection of disjoint simple polyhedra, $G$ is a graph, and $P \cap G$ is a finite set of nonvertices in $P$.

Definition. A simple polyhedron $P$ is special if it contains at least one vertex and if all its two-components are two-cells.

Note that there are finitely many special polyhedra with a given number of vertices.

Definition. A subpolyhedron $P \subset \stackrel{\circ}{M}$ of a compact three-manifold $M$ with nonempty boundary is said to be its spine if $M$ collapses to $P$ or, equivalently, if $M \backslash P$ is homeomorphic to $\partial M \times(0,1]$.

The spine is called almost special or special if it is a polyhedron of the corresponding type.
We will always assume that an almost special spine cannot be collapsed onto a proper subpolyhedron.

By a spine of a closed manifold $M$ we mean a spine of $M \backslash \stackrel{\circ}{D}^{3}$, where $D^{3}$ is an embedded closed ball in $M$. It is known that any compact threemanifold possesses an almost special (even a special) spine. Moreover, one can easily construct a special spine of $M$ starting from practically any presentation of $M$ [Matveev 1990]. Special spines possess an important property that favorably distinguishes them from simple and almost special spines: a three-manifold can be uniquely recovered from its special spine. Note that special spines can be considered as combinatorial objects and admit presentations in computer's memory as strings of integers that show how two-cells are attached to singular graphs of spines. To present a manifold by its almost special spine, additional information is needed on the way the spine should be thickened to become the three-manifold.

\section{SIMPLIFYING MOVES ON SPINES}

In what follows we will consider compact orientable three-manifolds whose boundaries are either empty or consist of spheres and tori.

We introduce six types of moves on almost special spines, each of which does not increase the number of vertices of the spine, and quite often decreases it. We call them simplifying moves. The moves transform not only spines, but may also transform the corresponding manifolds. Therefore, one should keep in memory additional information sufficient for recovering the original manifolds from the new ones.

Let $P$ be an almost special spine of a three-manifold $M$.

Move 1 (Admissible disc replacement). Let $P$ be a simple polyhedron. Suppose $D^{2}$ is a disc in $M$ such that $D^{2} \cap P=\partial D^{2}$ and that the curve $\partial D^{2}$ is in general position in $P$. Then $D^{2}$ cuts off $M \backslash P$ a ball $B^{3}$. Let $\alpha \neq D^{2}$ be a two-component of the simple polyhedron $P \cup D^{2}$ such that $\alpha$ separates $B^{3}$ 


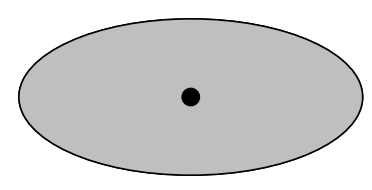

nonsingular point

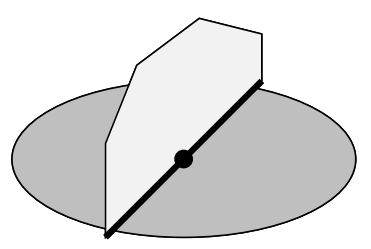

triple line

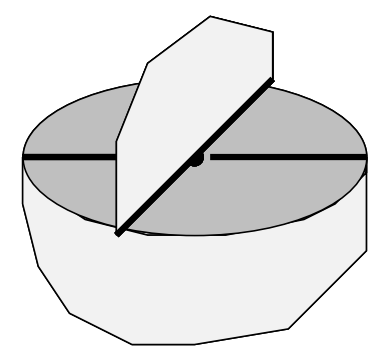

vertex

FIGURE 1. Each point in a simple polyhedron has a neighborhood that is topologically the cone over a circle (left), a circle with a diameter (middle), or a circle with three radii (right).

from $M \backslash B^{3}$. Removing from $\alpha$ an open two-disc and collapsing the resulting polyhedron as long as possible, we get another almost special spine $P_{1}$ of $M$. (See Figure 2.) We say that $P_{1}$ is obtained from $P$ by a disc replacement move.

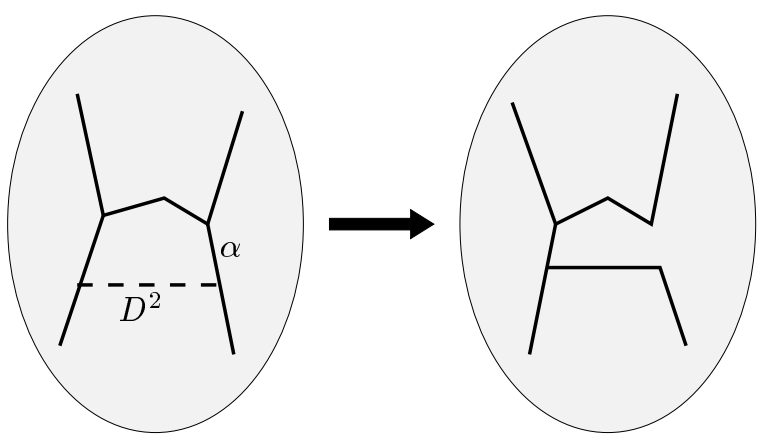

FIGURE 2. A disc replacement move.

A disc replacement move is called admissible if the following conditions hold:

(1) The move does not increase the number $v(P)$ of vertices of $P$; that is, $v\left(P_{1}\right) \leq v(P)$.

(2) $v\left(P \cup D^{2}\right)-v(P) \leq 4$.

An admissible disc replacement move is monotone if $v\left(P_{1}\right)<v(P)$, and horizontal if $v\left(P_{1}\right)=v(P)$.

Remark 2.1. It is easy to see that, applying to a special spine admissible disc replacement moves, one can get only finitely many different special spines.

Remark 2.2. Here is an important particular case of Move 1. Suppose a two-component $\alpha \subset P$ contains a nontrivial orientation-preserving simple closed curve $l$. Then there is a proper annulus $A \subset M$ such that $A \cap P=l$. Suppose that at least one of the boundary circles of $A$ bounds a disc in $\partial M$. Then there is a disc $D^{2}$ in $M$ such that $D^{2} \cap P=\partial D^{2}=l$, and we can simplify $P$ by Move 1.

Move 2 (Cutting of a two-component along a nontrivial circle). Suppose that a two-component $\alpha \subset P$ contains a nontrivial orientation-preserving simple closed curve $l$, and let $A \subset M$, with $A \cap P=l$, be a transverse proper annulus. Assume the boundary circles of $A$ are nontrivial in $\partial M$. Cutting $P$ along $l$ and collapsing the resulting polyhedron as long as possible, we get an almost special polyhedron $P_{1} \subset M$.

Denote by $L$ the connected component of $A \cup \partial M$ containing $A$. Let $Y^{3}$ be a regular neighborhood of $L$ in $M$ such that $P_{1}$ is a spine of $M_{1}=\overline{M \backslash Y^{3}}$. Then $M=M_{1} \cup Y^{3}$ and $M_{1} \cap Y^{3}$ consists of one or two tori. Note that, since the boundary circles of $A$ are nontrivial in $\partial M, L$ fibers onto circles. It follows that $Y^{3}$ is a circle bundle over a surface $F$. It is easy to see that $\chi(F)=-1$ and $\partial F$ consists of two or three circles. Therefore $F$ is homeomorphic either to a twice punctured disc $N^{2}$ or to a once punctured Möbius band $B^{2}$ (the latter happens when the coherently oriented boundary circles of $A$ lie on the same boundary torus of $M$ and have opposite orientations there). We may conclude that $Y^{3}=N^{2} \times S^{1}$ or $Y^{3}=B^{2} \tilde{\times} S^{1}$.

Moves 1 and 2 are basic ones. Applying them, we destroy two-components that contain nontrivial simple closed curves. As a byproduct, we may obtain almost special spines with one-dimensional 


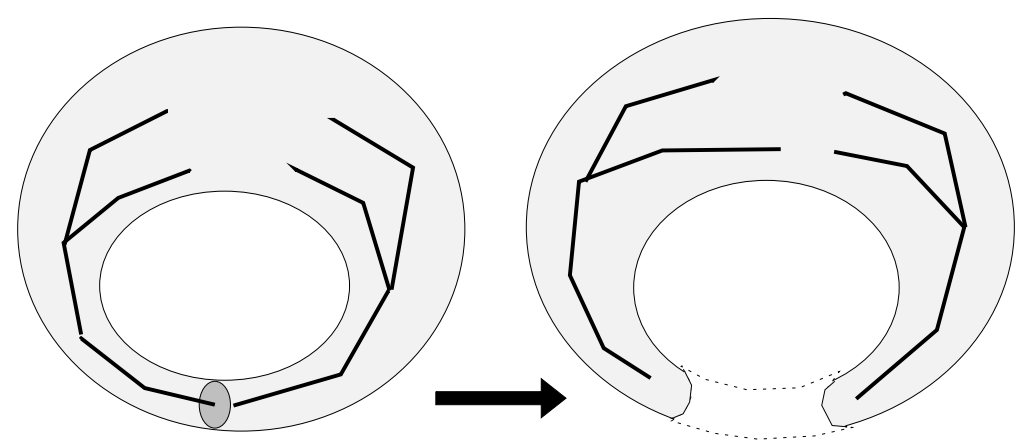

FIGURE 3. Cutting free arcs.

parts as well as spines of three-manifolds with several spherical boundary components. To simplify them, we use additional moves.

Move 3 (Cutting free arcs). Suppose that $P$ contains an arc $l$ such that no two-dimensional sheets are attached to $l$. Removing the interior of $l$ from $P$ and collapsing the resulting polyhedron as long as possible, we get an almost special polyhedron $P_{1} \subset P$.

To describe the corresponding transformation of $M$, denote by $D^{2}$ a proper disc in $M$ such that $D^{2}$ intersects $l$ transversely at exactly one point. Then $P_{1}$ is an almost special spine of a manifold $M_{1}$ that is obtained from $M$ by cutting along $D^{2}$. In other words, $M$ can be obtained from the new manifold $M_{1}$ by attaching handle of index 1 ; see Figure 3 . There are three cases.

(A) $M=\left(M_{1}+D^{3}\right) \#\left(D^{2} \times S^{1}\right)$, if $\partial D^{2}$ does not separate $\partial M$ (here " $+D^{3}$ " means that we fill up a spherical component of the boundary with a three-dimensional ball).

(B) $M=\left(M_{1}+D^{3}\right) \#\left(S^{2} \times S^{1}\right)$, if $D^{2}$ does not separate $M$ but $\partial D^{2}$ separates $\partial M$.

(C) $M=M_{1}^{\prime} \#\left(M_{1}^{\prime \prime}+D^{3}\right)$, if $D^{2}$ separates $M$, where $M_{1}^{\prime}, M_{1}^{\prime \prime}$ are the connected components of $M_{1}$.

Move 4 (Delicate piercing). Suppose that $\partial M$ consists of at least two components, and at least one of them is spherical. Then one can find a proper arc $l \subset M$ such that $A=l \cap P$ is a nonsingular point of $P$ and $l$ joints a spherical component of $\partial M$ with another one. Removing from $P$ an open disc neighborhood of $A$ and collapsing the resulting polyhedron as long as possible, we get an almost special spine $P_{1}$ of $M_{1}=M+D^{3}$. We call the piercing delicate since it induces a very mild modification of $M$.

Move 5 (Removing isolated points). If $P$ contains isolated points, remove them.

Move 6 (Rough piercing). Let $P$ be a special spine of a closed manifold $M$. Choose a two-component $\alpha$ of $P$ such that after removing $\stackrel{\circ}{\alpha}$ from $P$ and collapsing the resulting polyhedron, we get an almost special polyhedron $P_{1} \subset M$ with the smallest possible number of vertices. Then $P_{1}$ is an almost special spine of a new manifold $M_{1} \subset M$ such that $\partial M_{1}$ is a torus. Clearly, $M \backslash M_{1}$ is a solid torus; that is, $M$ is obtained from $M_{1}$ by a Dehn filling.

\section{EXPERIMENTAL RESULTS AND CONJECTURES}

We recall the following notion, which is naturally related to practically all the known methods of presenting manifolds and captures well the informal idea of complexity of three-manifolds [Matveev 1990].

Definition. A compact three-manifold $M$ has complexity $c(M)=k$ if $M$ possesses an almost special spine with $k$ vertices and admits no almost special spines with a smaller number of vertices.

The complexity possesses the following properties:

(1) For any integer $k$, there exist only finitely many distinct closed irreducible orientable three-manifolds of complexity $k$. 
(2) The complexity of the connected sum of threemanifolds is equal to the sum of their complexities, and the same is true for boundary connected sums.

(3) Let $M_{F}$ be obtained from a three-manifold $M$ by cutting along a proper incompressible surface $F \subset M$. Then $c\left(M_{F}\right) \leq c(M)$.

Remark 3.1. Using Moves 1, 3, 4, and 5, one can easily prove that any minimal almost special spine of a closed orientable irreducible three-manifold $M$ with $c(M)>0$ is a special one. There are exactly three closed orientable irreducible threemanifolds of complexity $0: S^{3}, R P^{3}$, and the lens space $L(3,1)$. Their minimal almost special spines (a point, $R P^{2}$, and a simple polyhedron without vertices with exactly one two-component, which is homeomorphic to the disc) are not special.

Theorem 3.2. All closed orientable three-manifolds of complexity at most 8 are graph-manifolds.

This was initially proved by computer; later, a purely theoretical proof was found [Matveev 1990]. We describe the main steps of the computer program used in the original proof.

Step 1. The program enumerates all the finitely many special polyhedra with at most 8 vertices.

Step 2. The program selects spines of closed orientable three-manifolds. (It is easy to decide if a special polyhedron is a spine of a three-manifold, and if it is, then the three-manifold is unique.)

Step 3. Then it tries to apply to each spine admissible disc replacement moves that decrease the number of vertices. If such a move is possible, then the corresponding three-manifold $M$ is not interesting for either it has a smaller complexity (and we have met it earlier), or it is a connected sum of closed manifolds of smaller complexities. Otherwise, we go to the next step.

Step 4. The program applies Move 6 (rough piercing) and simplifies the new spine by Moves $1-5$. Note that Move 6 is allowed only if the manifold is closed or has a spherical boundary, and it produces a manifold with a torus boundary.

The main observation resulting from the computer experiment is that if we start with a special spine of a closed orientable manifold $M$ with at most 8 vertices, then after Moves $1-6$ we always get the empty set. This means that $M$ is a graph-manifold.

Theorem 3.2 is sharp in the sense that there exist closed orientable three-manifolds of complexity 9 that are hyperbolic and therefore are not graphmanifolds. One of them has volume $0.94 \ldots$; this is the smallest known value for the volume of a closed orientable hyperbolic three-manifold [Weeks 1985; Matveev and Fomenko 1988].

We say that an almost special spine of a threemanifold $M$ is minimal if it has the smallest possible number of vertices.

Theorem 3.3. If a special spine of a closed orientable three-manifold $M$ contains less than 8 vertices and is not minimal, then it can be simplified by a monotone admissible disc replacement move. Any two minimal special spines of $M$ are related by horizontal admissible disc replacement moves.

This theorem is an experimental fact; it has been verified by a computer program.

The following conjectures have been motivated by the experimental results stated above, as well as by other observations.

Conjecture 3.4. If a special spine of a compact threemanifold is not minimal, then the number of its vertices can be decreased by admissible disc replacement moves.

If the conjecture is true, one can get a simple algorithm for recognition of the unknot, as follows. Apply to a spine of the knot complement admissible disc replacement moves as long as possible. The knot is trivial if and only if one eventually gets a circle. In the same way one would have a simple algorithm for recognition of the three-sphere.

Conjecture 3.4 may be too strong to be true, although vast computer experiments to find out a counterexample have been unsuccessful. This 
shows that, if exceptions exist, they are rare, and the above algorithms should give the circle or the point for a typical spine of the solid torus or the ball, respectively. Therefore, we have good practical partial procedures for recognizing the unknot and the sphere.

Conjecture 3.5. If a special spine of a closed graphmanifold is minimal, then any rough piercing move (Move 6) transforms it into an almost special spine of a graph-manifold.

The conjecture is true for all graph-manifolds up to complexity 8. It allows one to reduce the recognition problem for closed graph-manifolds to that for manifolds with boundaries.

\section{SPINES OF MANIFOLDS WITH ESSENTIAL ANNULI}

It is known [Matveev 1990] that if a three-manifold is reducible or boundary reducible, then its minimal almost special spine is not special. It turns out that the same is true for manifolds containing essential (that is, incompressible and boundary incompressible) annuli. This observation is especially important for the investigation of graphmanifolds with nonempty boundary, since all of them are reducible or boundary reducible or contain essential annuli.

Theorem 4.1. If a compact three-manifold $M$ contains an essential annulus, no minimal almost special spine of $M$ is special.

Remark 4.2. If an almost special spine is not special and has at least one vertex, it contains either a two-component not homeomorphic to the disc or one-dimensional part. Hence we can apply either Moves 1 and 2 or Move 3.

Before proving Theorem 4.1, we recall some notions of normal surface theory [Haken 1961]. Let $\xi$ be a handle decomposition of a three-manifold $M$ with nonempty boundary. It consists of handles of index 0,1 , and 2, called balls, beams, and plates, respectively. Connected components in the intersection of balls and beams are called islands; connected components in the intersection of balls and plates are called bridges. The boundaries of balls meet $\partial M$ along lakes. Any normal surface $F \subset M$ should intersect balls, beams and plates in a very specific way [Haken 1961]. In particular, the intersection of $F$ with balls should consist of elementary discs. The boundary curve $\partial E$ of each elementary disc $E$ should satisfy these conditions:

(1) The intersection of $\partial E$ with any bridge and any lake consists of no more than one segment.

(2) If $l$ is an arc in the intersection of $\partial E$ with a lake $L$ then the end points of $l$ should lie in different connected components of the intersection of $L$ with islands.

(3) If a lake and a bridge are adjacent then $\partial E$ intersects no more than one of them.

We say that an elementary disc $E$ has type $(m, n)$ if the circle $\partial E$ intersects $m$ bridges and $n$ lakes.

Any special spine $P$ of $M$ generates a handle decomposition $\xi_{P}$ of $M$. Balls, beams, and plates of the decomposition correspond respectively to vertices, edges, and two-components of $P$. The boundary of each ball contains exactly four islands, and any two of them are joined by exactly one bridge. See Figure 4.

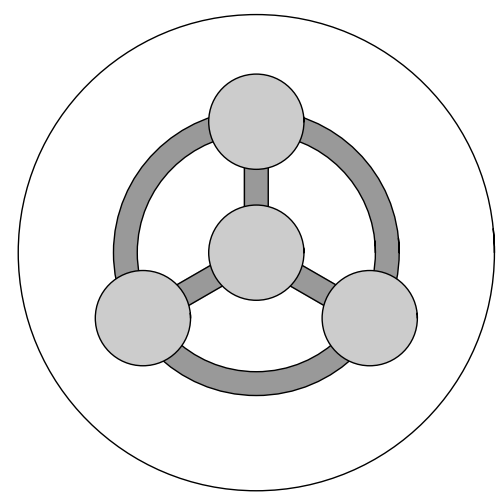

FIGURE 4. Boundary of a ball in a handle decomposition $\xi_{P}$.

It is not hard to see that any elementary disc for $\xi_{P}$ has one of the following types: $(4,0),(3,0)$, $(2,1),(1,2),(0,2),(0,3),(0,4)$. Each type determines the corresponding elementary disc in a 
unique way (up to homeomorphisms of the ball taking islands to islands, bridges to bridges, and lakes to lakes), except the type $(0,3)$, which determines two elementary discs.

For any beam $D^{2} \times I$ (with $D^{2} \times\{0\}$ and $D^{2} \times\{1\}$ being islands), the disc $D^{2} \times\{1 / 2\}$ is called the transverse disc of the beam.

Definition. Let $A$ be a proper annulus in a threemanifold $M$ with a special spine $P$ such that $A$ is normal with respect to $\xi_{P}$. We say that $A$ has a tail if the intersection of $A$ with the transverse disc of a beam contains a proper arc $l$ such that the end points of $l$ lie in the same circle of $\partial A$. The arc $l$ cuts off $A$ a disc $D_{l}$. We call $D_{l}$ a tail of $A$.

Lemma 4.3. If the handle decomposition $\xi_{P}$ generated by a special spine $P$ of a three-manifold $M$ contains a normal annulus $A$ with a tail $D_{l}$, then $P$ is not minimal.

Proof. Denote by $M_{D_{l}}$ the three-manifold obtained from $M$ by cutting along $D_{l}$. Evidently, $M_{D_{l}}$ is homeomorphic with $M$. The tail decomposes the balls of $\xi_{P}$ into balls, plates into plates, and beams into beams except the beam $B_{0}$ containing $l$. Coherently collapsing new balls, beams, and plates onto two-dimensional subsets, we get an almost special spine $P^{\prime}$ of $M_{D_{l}}$. Since each ball of $\xi_{P}$ contains no more than one vertex of $P^{\prime}$, we have $v\left(P^{\prime}\right) \leq v(P)$, where $v(P)$ denotes the number of vertices. Note that $P^{\prime}$ has a free edge arising from cutting and collapsing the beam $B_{0}$; see Figure 5 . After collapsing $P^{\prime}$ through this free edge, we get an almost special spine of $M_{D_{l}}$ with a smaller number of vertices.

Proof of Theorem 4.1. Let $P$ be a special spine of a three-manifold $M$ with an essential annulus. Since the annulus is incompressible and boundary incompressible, one can replace it by an annulus $A$ that is normal with respect to the handle decomposition $\xi_{P}$ of $M$ generated by $P$. If $A$ has a tail, then we apply Lemma 4.3 to find a simpler spine of $M$. Assume that $A$ has no tails. Since each elementary disc of type $(0,3)$ or $(0,4)$ in $A$ would determine at

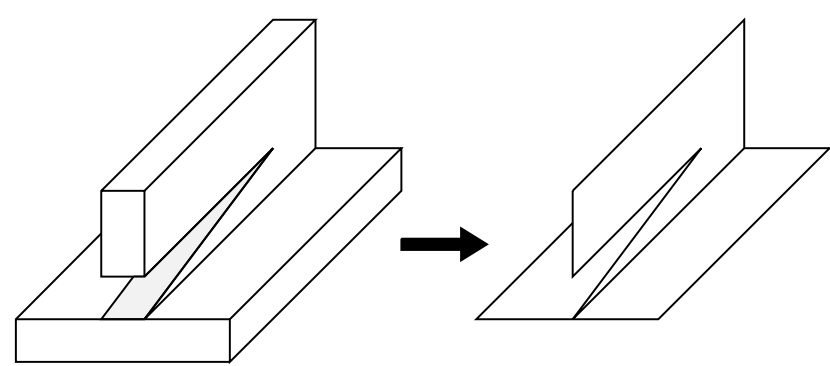

FIGURE 5. Result of cutting and collapsing of beam (see proof of Lemma 4.3).

least one tail, only types $(4,0),(3,0),(2,1),(1,2)$, $(0,2)$ for elementary discs in $A$ are possible. Moreover, if $E$ is an elementary disc of type $(1,2)$ or $(0,2)$, then two arcs in $\partial E \cap \partial M$ must lie in different components of $\partial A$.

Now cut $M$ along $A$ so that one component $S$ of $\partial A$ is preserved. In other words, we remove from $M$ the subset $S^{1} \times(0,1] \times \stackrel{\circ}{I}$, where $A=S^{1} \times[0,1]$ and $A \times I$ is a thin regular neighborhood of $A$ in $M$. As above, coherently collapsing the new balls, beams, and plates onto 2-dimensional subsets, we get an almost special spine $P^{\prime}$ of $M$ with $v\left(P^{\prime}\right) \leq$ $v(P)$. Moreover, if at least one elementary disc of type $(1,2)$ is present, then $v\left(P^{\prime}\right)<v(P)$. This is because each type $(1,2)$ elementary disc in the intersection of $A$ with a ball of $\xi_{P}$ annihilates the corresponding vertex of $P$; see Figure 6 .

We conclude the proof with the following remark: if there are no type $(1,2)$ elementary discs in $A$, then all elementary discs in $A$ have type $(0,2)$. In this case the core circle of $A$ can be shifted into a two-component of $P$. Since all two-components of $P$ are two-cells, it implies that the core circle is contractible, which contradicts the assumption that $A$ is incompressible.

\section{THE ALGORITHM}

Let $M$ be a compact three-manifold such that $\partial M$ is either empty or consists of tori. Our goal is to decompose $M$ into geometric pieces: in particular, to determine whether $M$ is a graph-manifold.

Step 1. Construct a special spine $P$ of $M$. 

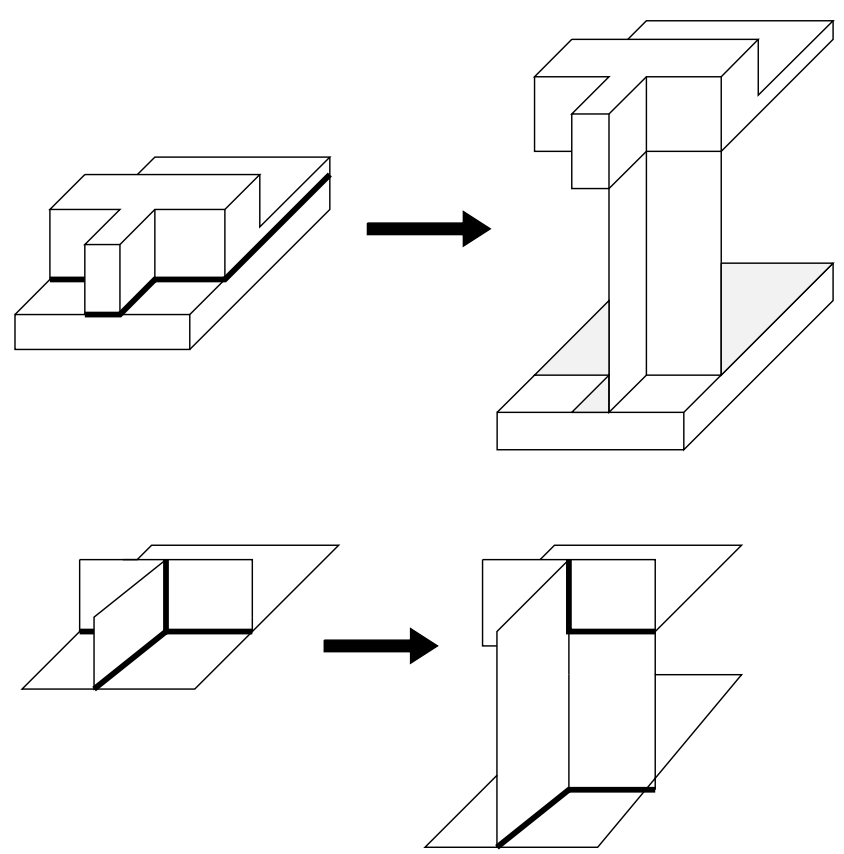

FIGURE 6. If an elementary disc of type $(1,2)$ is present, the number of vertices decreases (see proof of Theorem 4.1).

Step 2. Apply Moves $1-5$ to $P$ as long as possible. In the case of Move 2 when $N^{2} \times S^{1}$ or $B^{2} \tilde{\times} S^{1}$ is cut off we store the information on how this piece is attached to the remaining part of $M$. This can be done by selecting meridian-longitude pairs on boundary tori and controlling their behavior under further moves.

Step 3. In general, we get a collection of special spines. Then apply Move 6 to those of them that are spines of closed manifolds.

Step 4. Iterate Steps 2-3 as long as possible.

We get a collection $\mathscr{C}$ of special spines of manifolds such that the boundary of every manifold is nonempty and consists of tori.

Case 1: $\mathscr{C}$ is empty. This means that $M$ is a graphmanifold. We use the stored information to find out the canonical presentation of $M$ as a union of Seifert manifolds with explicitly given parameters and gluing matrices. The presentation distin- guishes the manifold from all other manifolds and can be considered as its name.

Case 2: $\mathscr{C}$ is a nonempty set of special spines. Then we get a decomposition of $M$ onto Seifert manifolds and unknown pieces that correspond to spines. Other methods should then be used to investigate the unknown manifolds; this is the reason why our algorithm is only a partial one. For example, one may test the manifolds for hyperbolicity by comparing with Jeff Weeks' table.

Remark 5.1. Recall that if $M$ is closed and its special spine $P$ contains at most 8 vertices, we always get Case 1.

Remark 5.2. If Conjectures 3.4 and 3.5 are true, then by Theorem 4.1 we get Case 1 if and only if $M$ is a graph-manifold.

\section{ACKNOWLEDGMENTS}

The paper is based on a talk at MSRI workshop on computational and algorithmic methods in threedimensional topology (March 10-14, 1997). The author wishes to thank MSRI for a friendly atmosphere and good conditions of work, and the referees for corrections and suggestions that have considerably improved a preliminary version of this paper.

\section{REFERENCES}

[Haken 1961] W. Haken, "Theorie der Normalfächen: Ein Isotopiekriterium für der Kreisknoten", Acta Math. 105 (1961), 245-375.

[Matveev 1990] S. V. Matveev, "Complexity theory of three-dimensional manifolds", Acta Appl. Math. 19:2 (1990), 101-130.

[Matveev and Fomenko 1988] S. V. Matveev and A. T. Fomenko, "Isoenergetic surfaces of Hamiltonian systems, the enumeration of three-dimensional manifolds in order of growth of their complexity, and the calculation of the volumes of closed hyperbolic manifolds", Uspekhi Mat. Nauk 43:1(259) (1988), 5-22, 247. In Russian; translation in Russian Math. Surveys 43:1 (1988), 3-24. 
[Scott 1983] P. Scott, "The geometries of 3-manifolds", Bull. London Math. Soc. 15:5 (1983), 401-487.

[Waldhausen 1967a] F. Waldhausen, "Eine Klasse von 3-dimensionalen Mannigfaltigkeiten, I", Invent. Math. 3 (1967), 308-333.
[Waldhausen 1967b] F. Waldhausen, "Eine Klasse von 3-dimensionalen Mannigfaltigkeiten, II", Invent. Math. 4 (1967), 87-117.

[Weeks 1985] J. R. Weeks, Hyperbolic structures on 3manifolds, Ph.D. thesis, Princeton University, 1985.

Sergei V. Matveev, Chelyabinsk State University, Kashirin Brothers Street, 129, Chelyabinsk, 454136, Russia (matveev@cgu.chel.su, matveev@csu.ac.ru)

Received March 31, 1997; accepted in revised form December 14, 1997 